\title{
Estudio de un brote intrahospitalario por Salmonella typhimurium productora de beta-lactamasa de espectro extendido SHV-5
}

\author{
Liliana Del Pozo ${ }^{1}$, Nazario Silva ${ }^{2}$, Augusto Valencia ${ }^{3}$, Javier Soto ${ }^{4}$, Juan C Riveros ${ }^{4}$, \\ Rosa Sacsaquispe ${ }^{5}$, Róger Calderón ${ }^{6}$, Víctor Suarez ${ }^{7}$
}

Resumen

\begin{abstract}
Antecedentes: Presencia de un brote intrahospitalario de Salmonella typhimurium productora de beta-lactamasas de espectro extendido ocurrido en el Hospital San Bartolome, entre el 17 de febrero y el 6 de marzo del año 2001. Objetivo: Identificar los mecanismos implicados en la transmisión de Salmonella typhimurium y caracterización de los genes asociados a la resistencia en beta-lactámicos. Diseño: Estudio clínico-bacteriológico retrospectivo. Lugar: Hospital Nacional Docente Madre Niño(Honadomani) San Bartolomé. Materiales biológicos: Aislamientos bacterianos provenientes de pacientes lactantes. Intervenciones: Se determinó la diversidad genética de cinco aislamientos bacterianos provenientes de pacientes lactantes hospitalizados en la Unidad pediátrica del hospital, utilizando REP-PCRy fingerprintplasmídico. Previamente, se caracterizóla resistencia antimicrobiana, determinando la presencia de beta-lactamasa de espectro extendido mediante la prueba de sinergia de doble disco; la variante fue identificada por PCR-secuenciamiento del gen bla ${ }^{\text {shv }}$. Principales medidas de resultados: Presencia de genotipos, plásmidos y beta-lactamasa de Salmonella typhimurium. Resultados: Se determinóla presencia de dos genotipos en los aislamientos de Salmonella typhimurium; el caso índice (sensible) presentóun genotipo diferente al de otros aislamientos resistentes pertenecientes a pacientes hospitalizados. Se determinóla presencia en S. typhimurium de un plásmido de peso molecular elevado de tamaño distinto a los de K. pneumoniae, pero probablemente relacionado con una cepa de E. coli intrahospitalaria. Se encontró la beta-lactamasa de espectro extendido SHV5 en los aislamientos de S. typhimuriumy E. coli. Conclusiones: El estudio sugiere que la diseminación de estas bacterias en los lactantes puede haber sido favorecida por varios factores que habrían intervenido en la transferencia de elementos genéticos responsables de la resistencia antimicrobiana.
\end{abstract}

Palabras clave Infección intrahospitalaria; infecciones por Salmonella; Salmonella typhimurium; beta-lactamasas.

Study of a hospital outbreak by enlarged SHV-5 spectrum beta-lactamase producing Salmonellatyphimurium

\section{Abstract}

Background: AnESBL Salmonellatyphimuriumoutbreakoccurred at San Bartolome hospital from February 17 through March 16,

\footnotetext{
1 Departamento de Pediatra, Hospital Nacional Docente Madre Niño San Bartolomé. Lima-Perú.

2 Departamento de Epidemiología, Hospital Nacional Docente Madre Niño San Bartolomé. Lima-Perú.

3 Departamento de Patología Clínica, Hospital Nacional Docente Madre Niño San Bartolomé. Lima-Perú.

4 Servicio de Microbiología Clínica, Hospital Nacional Docente Madre Niño San Bartolomé. Lima-Perú.

5 Laboratorio de IRAS e IIH, Instituto Nacional de Salud. Lima-Perú.

6 Laboratorio de Biotecnología y Biología Molecular, Instituto Nacional de Salud. Lima-Perú.

7 Infectología. Instituto Nacional de Salud. Lima-Perú.
}

2001. Objectives: To identify the mechanisminvolved in Salmonella typhimurium spread and genetic characterization of beta-lactamase resistance associated genes. Design: Clinical-bacteriologic retrospective study. Setting: San BartoloméMother Child Teaching National Hospital. Biologic materials: Bacterial isolations from lactating patients. Interventions: The genetic diversity was characterized from five bacterial isolates from infants admitted to the pediatric units, using REP-PCR and plasmid fingerprinting. We previously characterized the antimicrobial resistance, determining the presence of ESBL by the double disc diffusion method and the variant was identified by sequencing the gen bla ${ }^{\text {shv }}$. Main outcome measures: Salmonella typhimurium genotypes, plasmids and beta-lactamase presence. Results: We found two different genotypes among the Salmonella typhimurium isolates; the index case (susceptible) showed a different genotype and the other isolates coming from hospitalized children were resistant. One of the S. typhimurium plasmids had a heavier molecular 
weight than K. pneumoniae's but as heavy as the hospital acquired E. coli isolates plasmids. We found the ESBL SHV-5 in both S. typhimuriumy E. coli isolates. Conclusions: This report suggests that the bacteria spread among infants could be facilitated by many factors playing differentroles in the genetic material transfer responsible of the microbial resistance.

Key words: Cross infection; Salmonella infections; Salmonella typhimurium, beta-lactamases.

\section{INTRODUCCIÓN}

Las infecciones gastrointestinales siguen siendo un problema de salud pública mundial y entre los agentes patógenos responsables la Salmonella spp. es frecuente. Éste es un bacilo gram negativo que causa enfermedad diarreica bacteriana, como resultado de ingerir alimentos contaminados, particularmente los de origen animal $\left({ }^{1}\right)$.

Aunque no es recomendable tratar con antibióticos las gastroenteritis producidas por este microorganismo, la medicación tiene indicaciones específicas para las complicaciones invasoras, tales como bacteriemias, sepsis, meningitis y otras $\left({ }^{1,2}\right)$.

El uso inapropiado de antibióticos, de manera profiláctica, terapéutica y como promotores de crecimientos en granjas y alimentos de animales, es un factor importante en el desarrollo de resistencia bacteriana por patógenos $\left(^{3}\right)$. El constante uso inapropiado de antibióticos selecciona bacterias multiresistentes, así como productoras de beta-lactamasas de espectro extendido (BLEE), lo cual facilita su transmisión entre pacientes hospitalizados. Consecuentemente, la microflora multiresistente, mayormente compuesta de colonizadores comunes humanos, aparece en los hospitales. Como un género patógeno en humanos, Salmonella no es un miembro típico de esta microflora hospitalaria. Por consiguiente, la resistencia múltiple a los antibióticos está raramente asociada a este género. Así también, la detección de la BLEE en Salmonella es un fenómeno raro que se ha incrementado en los años recientes $\left({ }^{4}\right)$. Si Salmonella typhimurium puede existir el tiempo suficiente en la microflora hospitalaria, adquirirá e intercambiará plásmidos de resistencia múltiple con otros géneros bacterianos.

La producción de BLEEs es un mecanismo de resistencia de las bacterias gram negativas y derivan de las beta-lactamasas de amplio espectro TEM y SHV $\left({ }^{5}\right)$. Estas enzimas confieren resistencia a todas los oxi-imino-cefalosporinas, inactivando así penicilinas, monobactamos y cefalosporinas de primera, segunda, tercera y cuarta generación. Son frecuentemente codificadas por grandes plásmidos o elementos móviles que transportan múltiples determinantes de resistencia a antibióticos y se propagan entre los miembros de la familia Enterobacteriaceae $\left({ }^{1}\right)$.

La resistencia en cepas de Salmonella no typhi emerge en los '90s y rápidamente se han diseminado. Las primeras cepas fueron detectadas en Francia, en los años 1984 y 1987 (S. typhimurium SHV-2); esto fue seguido por la detección de otras cepas en Túnez (Salmonella wien SHV-2, 1988), Martinica ( $S$. panama TEM-3, en 1989), Francia ( $S$. kdougou TEM-3, en 1989), Argelia ( $S$. ambondaka CTX-2, en 1990), Argentina ( $S$. typhimurium CTX-M2, en 1991), España ( $S$. othmarsschen TEM-27, en 1995) y Polonia ( $S$. enteritidis CTX-M3, en 2002) ${ }^{(2,6)}$. Por lo expuesto, Salmonella puede expresar una variedad de tipo de BLEE, incluyendo TEM, SHV, PER, y CTX-M $\left({ }^{4,5,7}\right)$.

En el presente trabajo, se ha analizado un brote intrahospitalario producido por Salmonella typhimurium productora de la BLEE SHV-5, en la sala de lactantes del Hospital Nacional Docente Madre Niño San Bartolome, para determinar si los casos fueron contaminados e infectados por una única cepa que adquirió resistencia, dado que el primer aislamiento fue sensible a los antibióticos, mientras que los posteriores aislamiento del mismo paciente presentaron 
resistencia a los antibióticos probados. Para evaluar si los aislamientos del brote corresponden a una misma variante, se analizó el ADN genómico mediante el uso de fingerprint genómico y plasmídico $\left({ }^{8}\right)$, así como se determinó la variante genética de la BLEE.

\section{MATERIALES Y MÉTODOS}

El brote ocurrió en la unidad de terapia intensiva (UTI) pediátrica y en la sala de hospitalización de lactantes, del Departamento de Pediatría del Hospital San Bartolomé. Este hospital está ubicado en Lima Cercado, brinda los servicios de atención médica y quirúrgica dirigidos principalmente a la población infantil, donde la UTI cuenta con 4 camas, 3 ventiladores y en la sala de lactantes cuenta con 3 salas y 28 cunas.

Se obtuvo los datos clínicos obtenidos a partir de las historias clínicas de los pacientes hospitalizados en el momento del brote, que presentaron diarrea o septicemia, en marzo 2001, en el servicio de Pediatría del Hospital San Bartolomé. Este brote fue reportado por el Servicio de Microbiología, al detectarse la presencia de Salmonella typhimurium productora de BLEE.

La investigación ha determinado que el caso índice ingresó al hospital por el Servicio de Emergencia, el 17 de febrero de 2001, presentando un coprocultivo y hemocultivo positivos a $S$. typhimurium (aislamiento 01B101), sensible a todos los antibióticos. Posteriormente, a su ingreso en la sala de hospitalización de lactantes y por su evolución tórpida, nuevos coprocultivo y hemocultivo resultaron positivos a $S$. typhimurium resistente a beta-lactámicos, por producir BLEE (aislamiento 01D-200).

El segundo caso reingresa a sala de lactantes el día 1 de marzo de 2001, por diarrea aguda infecciosa, a los 2 días del alta, y cuyo coprocultivo y hemocultivo resultaron positivos a $S$. typhimurium productora de BLEE resistente a beta-lactámicos (aislamientos 01B-126 y 01B-221).

La epidemia fue controlada a principios del mes de marzo del mismo año, después del aislamiento de los pacientes con medidas de barrera de contactos.

Se tomó además muestras de otros pacientes, obteniendo hemocultivos positivos a Klebsiella pneumoniae en el momento del brote en la UTI y una muestra de coprocultivo positivo de otro paciente de la sala de hospitalización en lactantes durante el brote, resultó positivo a E. coli, productor de BLEE.

Todas las cepas fueron enviadas al Instituto de Salud, para los análisis de fingerprinting y caracterización de la betalactamasa de espectro extendido.

El aislamiento y la identificación inicial de las bacterias fueron realizadas con las técnicas bacteriológicas convencionales $\left({ }^{9,10}\right)$ y confirmadas mediante el sistema de identificación API 20E.

El análisis de la susceptibilidad antimicrobiana se realizó mediante las pruebas de sensibilidad y paneles de Microscan, y utilizando los antibióticos: ampicilina, amoxicilina/ácido clavulánico, cefalotina, cefuroxima, cefoxitina, cefotaxima, cefotaxima/ ácido clavulánico, ceftazidima, ceftazidima/ ácido clavulánico, aztreonam, cefepima, imipenem, meropenen, amikacina, ciprofloxacina, gentamicina, sulfametoxazol/ trimetropim, piperacilina, piperacilina/ tazobactam.

La confirmación de la producción de BLEE fue realizada mediante la prueba de sinergismo de doble disco, con cefotaxima/ ácido clavulánico y ceftazidima/ ácido clavulánico $\left({ }^{11}\right)$, siguiendo las recomendaciones de la National Commitee for Clinical Laboratory Standards -NCCLS ${ }^{12}$ ) (Tabla 1). 
Estudio de un brote intrahospitalario por Salmonella typhimurium

Tabla 1. Cultivo y antibiograma de Salmonella typhimurium.

\begin{tabular}{|c|c|c|c|c|c|}
\hline \multirow{2}{*}{$\begin{array}{l}\text { Caso } \\
\text { Tipo de muestra }\end{array}$} & \multicolumn{3}{|c|}{ Paciente 1} & \multicolumn{2}{|c|}{ Paciente 2} \\
\hline & Heces & Sangre & Sangre & Heces & Sangre \\
\hline Fecha & $17 / 02 / 2001$ & $17 / 02 / 2001$ & $23 / 02 / 2001$ & $01 / 03 / 2001$ & $01 / 03 / 2001$ \\
\hline $\mathrm{N}^{\circ}$ aislamiento & 01B-101 & 01D-178 & 01B-200 & 01B-126 & 01D-221 \\
\hline Ampicilina & $\mathrm{s}$ & & $\mathrm{R}$ & & $\mathrm{R}$ \\
\hline Amoxicilina/clavulonico & & $\mathrm{S}$ & $\mathrm{S}$ & I & $\mathrm{R}$ \\
\hline Cefalotina & $\mathrm{S}$ & $\mathrm{S}$ & & & \\
\hline Cefoxitina & & & & & $\mathrm{S}$ \\
\hline Ceftriaxona & $\mathrm{S}$ & $\mathrm{s}$ & $\mathrm{R}$ & $\mathrm{R}$ & $\mathrm{R}$ \\
\hline Ceftazidime & & $\mathrm{S}$ & $\mathrm{R}$ & $\mathrm{R}$ & $\mathrm{R}$ \\
\hline Imipenem & & $\mathrm{s}$ & $\mathrm{s}$ & $\mathrm{s}$ & $\mathrm{s}$ \\
\hline Aztreonam & & $\mathrm{S}$ & $\mathrm{R}$ & $\mathrm{R}$ & $\mathrm{R}$ \\
\hline Amikacina & $\mathrm{s}$ & $\mathrm{S}$ & $\mathrm{R}$ & $\mathrm{R}$ & $\mathrm{R}$ \\
\hline Gentamicina & $\mathrm{S}$ & & & $\mathrm{R}$ & \\
\hline Ciprofloxacina & $\mathrm{S}$ & $\mathrm{S}$ & $\mathrm{S}$ & $\mathrm{s}$ & $\mathrm{S}$ \\
\hline Ac.Nalidixico & $\mathrm{S}$ & & & $\mathrm{s}$ & \\
\hline Cloranfenicol & $\mathrm{S}$ & & $\mathrm{R}$ & $\mathrm{R}$ & $\mathrm{R}$ \\
\hline STX S & & $\mathrm{R}$ & $\mathrm{R}$ & $\mathrm{R}$ & \\
\hline Cefepime & & & & & $\mathrm{R}$ \\
\hline Amoxicilina & & & & $\mathrm{R}$ & \\
\hline Furazolidona & $\mathrm{S}$ & & & I & \\
\hline Cultivo positivo a: & S. typhimurium & S. typhimurium & S. typhimurium & S. typhimurium & S.typhimurium \\
\hline Sala de Hospitalization & UTI & UTI & Lactante & Emergencia & Lactante \\
\hline BLEE & - & - & + & + & + \\
\hline
\end{tabular}

El ADN plasmídico fue aislado siguiendo el protocolo descrito por Sambrook y col. $\left({ }^{13}\right)$, en el cual las bacterias fueron sometidas a una lisis alcalina con $\mathrm{NaOH}-\mathrm{SDS}$ y una precipitación con acetato de potasio. Posteriormente, el ADN fue precipitado con etanol y resuspendido en agua estéril. Los plásmidos extraídos fueron evaluados mediante electroforesis en agarosa al $1 \%$ y registrados fotográficamente (ver Figura 1).

El análisis de fingerprint genómico $\left({ }^{14}\right)$ fue realizado mediante REP-PCR $\left({ }^{15}\right)$, usando $50 \mathrm{ng}$ de ADN genómico purificado y los oligonucleótidos REP1R-DT y REP2-D (Integrated DNA Technologies Inc. Iowa). La mezcla de reacción en un volumen final de $25 \mu \mathrm{L}$ estuvo constituida por $0,25 \mathrm{mM}$ de desoxinucleótidos fosfato, $3 \mathrm{mM}$ de $\mathrm{MgCl}_{2} ; 1 \quad \mu \mathrm{M}$ de los oligonucleótidos REP1R-DT y REP2-D y 2 U de enzima Taq ADN polimerasa. Las temperaturas usadas para la amplificación fueron: $95{ }^{\circ} \mathrm{C}$ por 5 minutos y 10 ciclos de $94{ }^{\circ} \mathrm{C}$ durante 1 minuto, annealling $35{ }^{\circ} \mathrm{C}$ durante 2 minutos y extensión a $72{ }^{\circ} \mathrm{C}$ durante 1 minuto, con un ramp de 6 minutos entre el annealling y la extensión. Luego, 25 ciclos de una denaturación a $95{ }^{\circ} \mathrm{C}$, durante 1 minuto, annealling $50{ }^{\circ} \mathrm{C}$ durante 2 minutos y extensión a $72{ }^{\circ} \mathrm{C}$ durante 1 minuto, con un ramp de 4 minutos entre el annealling y la extensión. Finalmente, una extensión de 72 ${ }^{\circ} \mathrm{C}$ por 10 minutos $\left({ }^{13}\right)$. Los fragmentos amplificados fueron separados mediante electroforesis en agarosa al 1,5\%, teñidos con bromuro de etidio y observados en un transiluminador ultravioleta. Los perfiles fueron registrados fotográficamente y escaneados para el análisis (Figura 2).

La relación entre los perfiles de fingerprint fue calculada usando el coeficiente de Dice $\left({ }^{14}\right)$, usando el programa Gel Compar II v2.5 


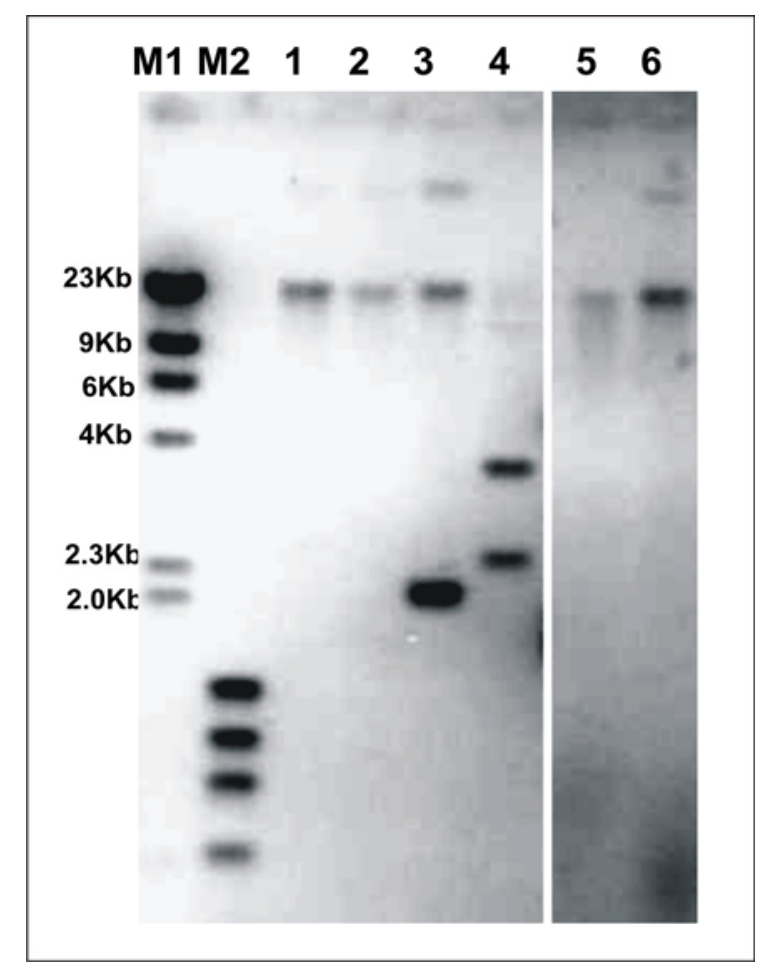

Figura 1. Patrones de ADN plasmídico en aislamientos intrahospitalarios de Salmonella typhimurium y Escherichia coli. Electroforesis en agarosa al 1\%, conteniendo los patrones de ADN plasmídico de Salmonella typhimurium y K. pneumoniae. El ADN plasmídico fue extraído mediante lisis alcalina y $10 \mathrm{uL}$ de plásmidos, pertenecientes a cada aislamiento, fueron sembrados en el gel de electroforesis. M1: Marcador Lambda Hind III, M2 100 pb Ladder. 1: S. typhimurium 126, 2: S. typhimurium 192, 3: S. typhimurium 200, 4: S. typhimurium 221. 5: S. typhimurium 101. 6: E. coli 138.

1998 (Applied Maths, Bélgica), basando el análisis en el algoritmo UPGMA 10, con una optimización y tolerancia en la posición de bandas de $1 \%$ (ver Figura 3), lo cual es esquematizado en un cladograma.

Los genes $b l a^{s h v}$ íntegros fueron amplificados mediante PCR, usando oligonucleólidos referenciales $\left({ }^{16}\right)$ y $50 \mathrm{ng}$ de ADN genómico de cada aislamiento como molde. Luego, los productos fueron purificados mediante el uso de columnas de sílica del QIAQuick PCR Purification Kit (QIAGEN). Después, la secuencia nucleotídica de los genes amplificados por
PCR fueron determinados en el secuenciador automático ALF Express de Amersham Pharmacia Biotech, del Laboratorio de Biotecnología y Biología Molecular del Instituto Nacional de Salud. Y, finalmente, fueron interpretados en el software ALFWin ${ }^{\mathrm{TM}}$ Sequence Analyser 2.0, 1998 y se utilizó el software NCBI Blast.

\section{RESULTADOS}

El 17 de febrero de 2001, se presentó en el servicio de emergencia el caso de un lactante con diarrea aguda infecciosa bacteriana, deshidratación severa y desnutrición crónica. Este paciente recibió hidratación, administrándosele cloranfenicol y amikacina y fue ingresado a la unidad de

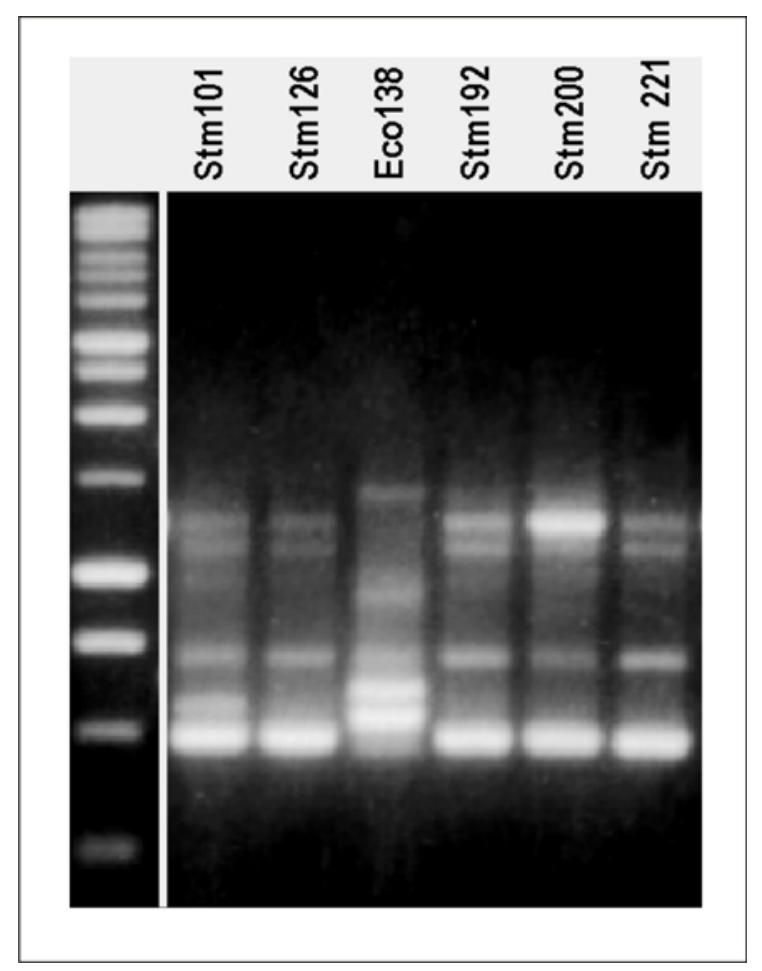

Figura 2. Patrones ribotípicos en aislamientos intrahospitalarios de Salmonella typhiumurium y E. coli. Foto que muestra los patrones de REP PCR de los aislamientos de Salmonella typhimurium y E. coli intrahospitalaria. M: Marcador 1 kb Ladder. Stm: Aislamientos de S. typhimurium. 
Dice (Tol 1,0\%-1,0\%) (H>0,0\% S>0,0\%) [0,0\%-100,0\%]

\section{KLEB SALM $0604 \quad$ KLEB SALM 0604}

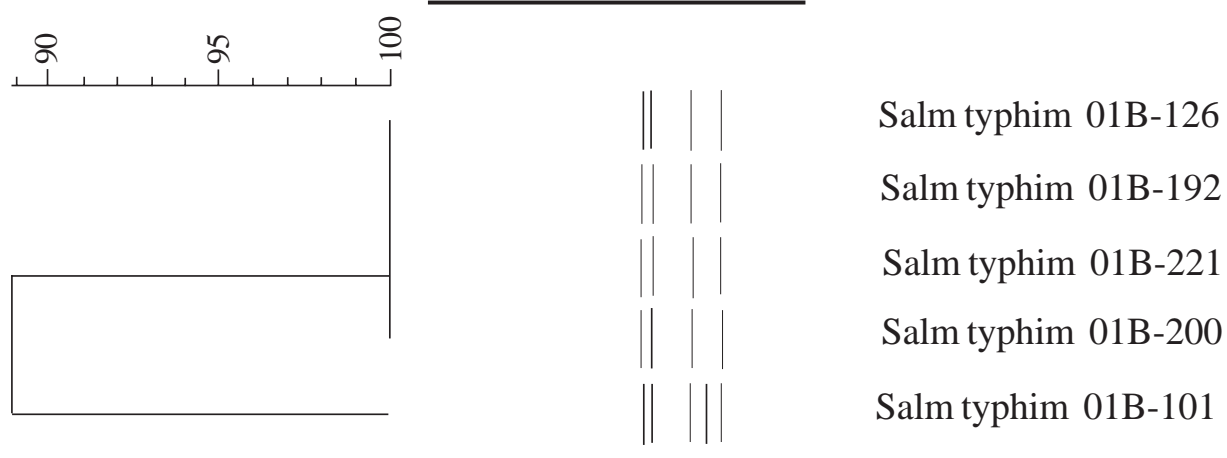

Figura 3. Árbol cladístico de genotipos obtenidos al analizar mediante REP PCR los aislamientos intrahospitalarios de Salmonella typhimurium involucrados en el brote intrahospitalario.

terapia intensiva, permaneciendo por 2 días, en donde le fueron tomados un hemocultivo y un coprocultivo, aislando Salmonella typhimurium susceptible a antibióticos (ver Tabla 1). Luego, fue derivado a la sala de lactante. Por la evolución tórpida, el paciente presentó un cuadro de deshidratación e hiponatremia, por cuadro de probable sepsis; se le cambió el tratamiento a ceftriaxona, previo hemocultivo y coprocultivo, aislando Salmonella typhimurium resistente a antibióticos y productora de BLEE. El tratamiento definitivo fue con ciprofloxacina y se procedió al aislamiento del paciente.

En la siguiente semana, el 21 de febrero de 2001, un nuevo paciente, lactante mujer de 8 meses, con diagnóstico de celulitis en miembros inferiores, eritema perineal,

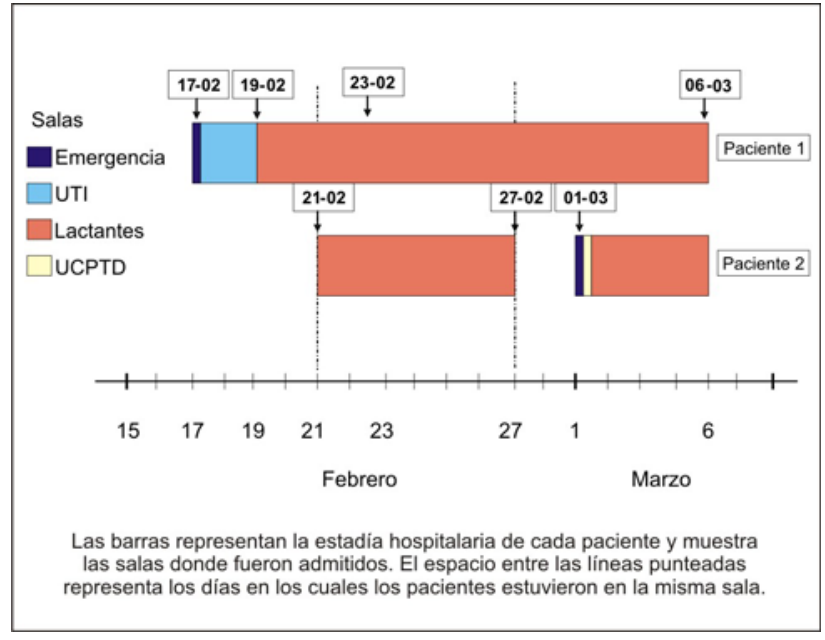

Figura 4. Estadía hospitalaria y traslado de los pacientes entre las salas

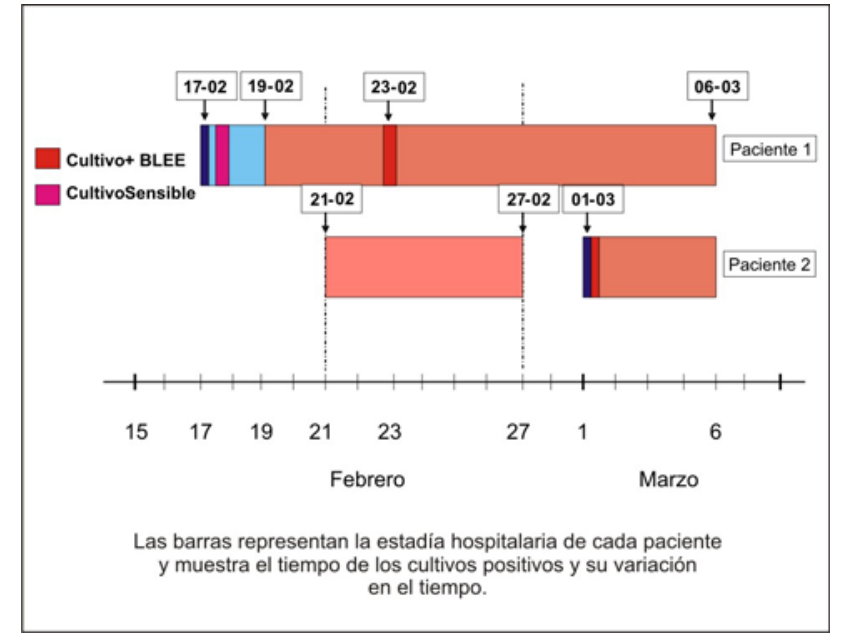

Figura 5. Momento del cultivo en la estadía hospitalaria de los pacientes, durante el brote. 
candidiasis, ingresa desde Emergencia a la sala de Lactante. El tratamiento recibido fue oxacilina y se le dio de alta el día 27 de febrero de 2001, reingresando el 1 de marzo de 2001 con el diagnóstico de deshidratación moderada por diarrea aguda infecciosa. Se le tomó cultivos, de sangre y heces, aislando en ambas muestras Salmonella typhimurium resistente a antibióticos y productoras de BLEE, por lo que se le inició tratamiento con ciprofloxacina y se procedió al aislamiento del paciente (Figuras 4 y 5).

Algunos hemocultivos de otros pacientes resultaron positivos a Klebsiella pneumoniae, simultáneamente, en el momento del brote en la UTI, así como un coprocultivo resultó positivo a Escherichia coli, proveniente de otro paciente en la sala de hospitalización de lactantes, durante el brote.

Todos los aislamientos de Salmonella typhimurium, excepto la primera, fueron productoras de BLEE.

Los aislamientos de $S$. typhimurium presentaron diferentes perfiles de plásmidos de peso molecular bajo. Sin embargo, los aislamientos resistentes presentaron un único plasmido de peso molecular elevado. Cabe señalar la similitud del tamaño mostrado por el plásmido de peso molecular elevado encontrado en la cepa de E. coli intrahospitalaria, aislada simultáneamente al brote (Figura 1).

Mediante el análisis de fingerprint genómico (REP PCR), se determinó que existen por lo menos dos genotipos entre los aislamientos de Salmonella typhimurium intrahospitalaria, siendo el aislamiento índice diferente a los otros casos.

El análisis de susceptibilidad mostró que los aislamientos fueron resistentes a la mayoría de los antibióticos beta-lactámicos, siendo susceptibles solo a ciprofloxacina e imipenem. La prueba de sinergia de doble disco determinó que eran productoras de beta-lactamasa de espectro extendido; determinándose por secuenciamiento del gen bla que pertenecían a la familia SHV, variante SHV-5.

\section{DISCUSIÓN}

El presente trabajo muestra la presencia de un brote intrahospitalario de diarrea aguda producida por Salmonella typhimurium, en nuestro hospital, confirmado por medio de los hemocultivos y coprocultivos, tanto en el caso índice como en los otros casos. Además, se demostró que este brote era producido por $S$. typhimurium productora de la BLEE SHV-5.

La primera Salmonella typhimurium productora de BLEE fue detectada en Francia, en 1984, y en América Latina fue recién detectada en Argentina, en $1991\left(^{6}\right)$. Los estudios realizados en Canadá en el año $2003\left({ }^{4}\right)$ describen a Salmonella typhimurium productora de BLEEs TEM-1 y SHV-2 ${ }^{\mathrm{a}}$.

Un estudio en Polonia (2002) determinó que Salmonella typhimurium productora de BLEE CTX-M3 y SHV-2 ${ }^{\text {a }}$ tiene una alta transmisión y se encuentra ampliamente diseminada en ese país $\left(^{5}\right)$.

Un reporte en Tucumán-Argentina analiza un brote de gastroenteritis por Salmonella typhimurium multirresistente, en población pediátrica, entre junio y noviembre 1991, y se observa resistencia a antibióticos, como ampicilina, cloranfenicol, TMP/sulfa, cefalosporinas de tercera generación y aminoglicósidos, excepto Netilmicina. Las alternativas terapéuticas fueron furazolidona, Colistín, imipenem y ciprofloxacina $\left({ }^{17}\right)$.

Otro reporte evalúa la transferencia de resistencia cefalosporina de tercera generación y de genes que codifican BLEE de cepas hospitalarias de Klebsiella neumoniae a otras enterobacterias, aislados en hospital de Chile, a través de métodos de detección de 
enzimas BLEE y detección de genes $b l a^{s h v-1}$, beta-lactamasa, por reacción de cadena de polimerasa. Los resultados en el estudio confirman que la Klebsiella ha sido catalogada como el principal reservorio del gen BLEE y otros genes de resistencia y evidencian la transferencia horizontal de genes de resistencia BLEE entre cepas de especies de bacilos Gram negativas $\left({ }^{18}\right)$.

En referencias de nuestro país $\left({ }^{19}\right)$, este es el primer reporte de Salmonella typhimurium productora de BLEE SHV-5 en Lima. Sin embargo, esta enzima ha sido anteriormente reportada en una UCI pediátrica de este mismo hospital, en aislamientos de Klebsiella pneumoniae y Enterobacter cloacae productora de beta-lactamasa de espectro extendido tipo SHV-5. Ello nos sugiere la posibilidad de transferencia de este genotipo a través de plásmidos y que han permanecido hasta la actualidad.

En otro estudio transversal y retrospectivo en el mismo hospital, cuyo objetivo fue investigar la presencia de portadores de Klebsiella pneumoniae productora de BLEE SHV-5, en neonatos de la UCI, se encontró que el $60 \%$ era portadores $\left({ }^{20}\right)$. Ello nos sugiere que existe una presencia endémica de esta bacteria productora de la enzima, reforzando la posibilidad de transferencia a partir de plásmidos codificantes de esta BLEE $\left.{ }^{(21}\right)$.

No se pudo demostrar que la Salmonella typhimurium del caso índice adquirió un plásmido de resistencia intrahospitalaria cuyas cepas aisladas de hemocultivos y coprocultivos iniciales fueron sensibles a todos los antibióticos y que luego, a partir del mismo paciente, se aisló una cepa de $S$. typhimurium multiresistente, sugiriendo la presencia de 2 cepas distintas.

Además, pensamos que el plásmido del aislamiento de $E$. coli podría contener la misma información determinante de la resistencia antimicrobiana, debido a que en esta bacteria también fue identificada la misma BLEE SHV-5, lo cual indica que podríamos tener fuentes de contaminación o reservorios en el hospital. Debido a ello, se debería incrementar los cuidados en la atención médica asistencial de los pacientes, especialmente en neonatos y pacientes inmunosuprimidos.

El estudio sugiere que la diseminación de estas bacterias en la sala de lactantes pudo haber sido favorecida por un inadecuado manejo asistencial, con la consiguiente transferencia de elementos genéticos responsables de la resistencia microbiana.

\section{AGRADECIMIENTOS}

Los autores expresan su agradecimiento al Técnico del Laboratorio de Microbiología del HONADOMANI San Bartolome Alfonso Terán Vásquez por su valiosa participación en el aislamiento de las cepas de S. typhimurium.

\section{REFERENCIAS BIBLIOGRÁFICAS}

1. Kaye K, Engemann J, Fraimow H, Abrutyn E. Pathogens resistant to antimicrobial agents: epidemiology, molecular mechanisms, and clinical management. Infect Dis Clin North Am. 2004;18(3):467-511.

2. Guzman-Blanco M, Casellas JM, Sader HS. Bacterial resistance to antimicrobial agents in Latin America. The giant is awakening. Infect Dis Clin North Am. 2000;14(1):67-81

3. Maguiña C. Infecciones nosocomiales [Editorial]. Diagnóstico. 2001;40(3)117-8.

4. Mulvey MR, Soule G, Boyd D, Demczuk W, Ahmed R; Multi-provincial Salmonella Typhimurium Case Control Study Group. Characterization of the first extended-spectrum betalactamase-producing Salmonella isolate identified in Canada. J Clin Microbiol. 2003;41(1):460-2.

5. Baraniak A, Sadowy E, Hryniewicz W, Gniadkowski M. Two different extended-spectrum beta-lactamases (ESBLs) in one of the first ESBL-producing salmonella isolates in Poland. J Clin Microbiol. 2002;40(3):1095-7.

6. Ait Mhand R, Soukri A, Amarouch H, El Mdaghri N, Benhachir M. Typing of enlarged-spectrum B-lactamaseproducing Salmonella typhimurium strains, isolated from a Pediatric Unit. Cahiers Sante. 1999;9(6):341-4.

7. AitMhand R, Soukri A, Moustaoui N, Amarouch H, ElMdaghri N, Sirot D, et al. Plasmid-mediated TEM-3 extended-spectrum beta-lactamase production in Salmonella 
typhimurium in Casablanca. J Antimicrob Chemother. 2002;49(1):169-72.

8. Instituto Nacional de Salud. Manual de procedimientos para la investigación de brotes de infecciones intrahospitalarias producidas por bacterias mediante métodos de biología molecular. Serie de normas Técnicas N 35. Lima: INS; 2002.

9. Sacsaquispe R, Ventura G. Manual y procedimientos bacteriológicos en infecciones intrahospitalarias. Series de normas técnicas N 28. Lima. INS, Ministerio de Salud; 2001.

10. Farmer J. Enterobacteriaceae: Introduction and Identification. En: Murray PR, Baron EJ, Pfaller Ma, Tenover FC, Yolken RH. Manual of Clinical Microbiology. 7th ed. Washington DC: American Society for Microbiology; 1999.

11. Javalier V, Nicolas MH, Fournier G, Philippon A. Extended broad-spectrum beta-lactamases conferring transferable resistance to newer beta-lactam agents in Enterobactereaceae: hospital prevalence and susceptibility patterns. Rev Infect Dis. 1988;10:867-78.

12. Ferraro M, Craig W, Dudley M, Eliopolus G, Hecht D, Hindler J, et al. Performance standards for antimicrobial disk suceptibility test: Approved Standard [document M2A7]. 7th ed. Pennsylvania: NCCLS; 2000.

13. Sambrook J, Fritsch EF, Maniatis T. Molecular Cloning. A Laboratory Manual. 2nd Edition. New York: Cold Spring Harbor Laboratory Press; 1989.

14. Popovic T, Bopp C, Olsvik, Kiehbaluch J. Ribotyping in molecular epidemiology 573. En: Persing DH, Smith TF, Tenover FC, White TJ (ed.). Diagnostic molecular microbiology: principles and applications. Washington, DC: American Society for Microbiology; 1993.

15. Tcherneva E, Rijpens N, Naydensky C, Herman L. Repetitive element sequence based polymerase chain reaction for typing of Brucella stain. Vet Microbial.1996;51 (1-2):169-78.
16. Arlet G, Philippon A. Construction by polymerase chain reaction and use of intragenic DNA probes for three main types of transferable $\beta$-lactamase (TEM, SHV, and CARB). FEMS Microbiol Lett. 1991;66:19-25. Erratum in: FEMS Microbiol Lett. 1991;68:125.

17. Silman M, Fernández N, Corti A, Argota C, Fernández SM, Assa J, et al. Brote intrahospitalario de salmonella thyphimurium multiresistente. Cienc Méd San Miguel de Tucumán. 1992;7(5):287-95.

18. Sánchez M, Bello H, Domínguez M, Mella S, Zemelman R, González G. Transferncia de b-lactamasa de espectro extendido desde cepas hospitalarias de Klebsiella pneumoniae a otras especies de enterobacterias. Rev Méd Chile. 2006;134:415-20.

19. Calderón R, Sacsaquispe R, Pasteran F, Galas M, Soto J, Riveros J, et al. Caracterización molecular de Klebsiella pneumoniae y Enterobacter cloacae productora de $\beta$ lactamasas de espectro extendido tipo SHV-5 en una unidad de cuidados intensivos neonatal de Lima. Rev Per Med Exp Salud Pública. 2003;20(3):121-7

20. Campos F, Soto J, Sacsaquispe R, Bazan C, Silva N. Klebsiella pneumoniae productora de $\beta$ Lactamasa de espectro extendido en una Unidad de Cuidado Intensivo Neonatal. Lima; 2003.

21. Fierer J, Guiney D. Extended-spectrum beta-lactamases: a plague of plasmids. JAMA. 1999;281(6):563-4.

Manuscrito recibido el 07 de agosto de 2006 y aceptado para publicación el 16 de octubre de 2006.

Correspondencia:

Dra. Liliana del Pozo

Correo-e: lilidelpozo@yahoo.es 